\title{
Spin polarization of the transport current at the free surface of bulk NiMnSb
}

\author{
S. K. Clowes, Y. Miyoshi, Y. Bugoslavsky, and W. R. Branford \\ Blackett Laboratory, Department of Physics, Imperial College, Prince Consort Rd, London SW7 2BZ, England \\ C. Grigorescu \\ National Institute for Optoelectronics, Bucharest, Romania \\ S. A. Manea \\ National Institute for Material Physics, Bucharest, Romania \\ O. Monnereau \\ Laboratoire MADIREL équipe Elaboration, UMR 6121 CNRS-Univ. de Provence, Marseille Cedex 20, France \\ L. F. Cohen \\ Blackett Laboratory, Department of Physics, Imperial College, Prince Consort Rd, London SW7 2BZ, England \\ (Received 2 October 2003; revised manuscript received 5 January 2004; published 24 June 2004)
}

\begin{abstract}
The point contact Andreev reflection employing niobium tips was used to determine the degree of transport current spin polarization $\left(P_{t}\right)$ at the free surface of bulk NiMnSb at $4.2 \mathrm{~K}$. The data was analyzed within the framework of a modified version of the Blonder, Tinkham, and Klapwijk formulism taking into account the two spin polarized channels in the ferromagnet and treating the interface as a planar delta function barrier of height $Z$ between free electron materials. We find that the measured spin polarization is rather insensitive to different surface preparations and magnetic domain structure, and the maximal value of the $P_{t}$ at $Z=0$ is $44 \%$, consistent with recent calculations of the surface reconstruction of NiMnSb. Saturation magnetization of the samples was found to be $3.6 \mu_{\mathrm{B}}$ per formula unit indicative of a small amount of atomic disorder.
\end{abstract}

DOI: 10.1103/PhysRevB.69.214425

PACS number(s): 72.25.-b, 74.50.+r

\section{INTRODUCTION}

Electronic devices whose functionality is determined by the orientation of electron spin are described as spintronic devices. ${ }^{1}$ One focus of this work has been the attempt to create a hybrid ferromagnet-semiconductor device based on the Datta and Das spin transistor. ${ }^{2,3}$ Significant progress has been made with all semiconductor systems using dilute magnetic semiconductors as the source of spin polarized current. In the case of BeMnZnSe, 90\% efficiency spin injection into GaAs has been observed, ${ }^{4}$ albeit below $5 \mathrm{~K}$ and in high magnetic fields. Spin injection from ferromagnetic metals into semiconductors requires a tunnel barrier ${ }^{5-7}$ to overcome the conductance mismatch problem. Indeed relatively efficient room temperature spin injection has been reported in permalloy-alumina-AlGaAs/GaAs light emitting diodes ${ }^{8}$ and permalloy/Schottky barrier/GaAs diodes ${ }^{9}$ demonstrating the possibility of a viable route to room temperature hybrid spin devices using these material systems.

Datta and Das originally proposed a spin transistor using Fe contacts on a narrow gap semiconductor. ${ }^{3} \mathrm{NiMnSb}$ should be a more suitable alternative to $\mathrm{Fe}$ as it is supposedly a half metallic ferromagnet ${ }^{10}$ with a Curie temperature of $728 \mathrm{~K} .{ }^{11}$ It also has only a $2.4 \%$ lattice mismatch with the narrow gap semiconductor InAs. No successful devices using NiMnSb has been reported to date and relatively few measurements of the polarization. Spin polarized photoemission measurements determine the bare polarization $(P)$ of the density of states at $E_{F}$ and $P$ at the free surface of NiMnSb have been reported to be about $50 \% .^{12}$ Planar $\mathrm{NiMnSb} / \mathrm{AlO}_{3} / \mathrm{Ni}_{20} \mathrm{Fe}_{80}$ tunnel structures were used ${ }^{13}$ to estimate the polarization of the tunnel current $P_{m}=28 \%$. Preliminary measurements of transport spin polarization of $\mathrm{NiMnSb}$ thin films using the point contact Andreev reflection (PCAR) technique estimate $P_{i}=58 \% .{ }^{14}$ A more recent study using PCAR has reported a value of $\sim 45 \%$ for single crystal NiMnSb. ${ }^{15}$ The latter measurement was part of the initial demonstration study of the PCAR technique and $P_{t}$ was determined using a crude method valid only for a clean interface between the superconducting point contact and the metal surface.

In the current paper, we have used the PCAR technique to explore the transport spin polarization of bulk NiMnSb that is to be used as a target for pulsed laser deposition. The data has been analyzed in terms of the extension to the Blonder, Tinkham, and Klapwijk (BTK) theory developed by Mazin and co-workers. ${ }^{16}$ This model has been applied previously by other groups to analyze the transport polarization of $\mathrm{CrO}_{2},{ }^{17}$ $\mathrm{SrRuO}_{3},{ }^{18,19}$ and $\mathrm{La}_{1-x} \mathrm{Sr}_{x} \mathrm{MnO}_{3} .{ }^{20}$ Recently it has been shown that this approach may have limitations for certain material systems because the reflection of conduction electrons at an interface between a nonmagnetic metal and ferromagnetic metal can be strongly spin dependent. ${ }^{21,22}$ There is also the problem that the model does not take into account spin dependent transport across the barrier. ${ }^{14,16}$ However experimental conductance spectra fit extremely well to the extended BTK model. It is believed that this is due to the dimensionless barrier parameter $(Z)$ containing more physics than simply the strength of the $\delta$-function barrier. ${ }^{23}$ In the case of significant spin dependent reflection due to a mismatch of the Fermi velocities it should not be possible to 


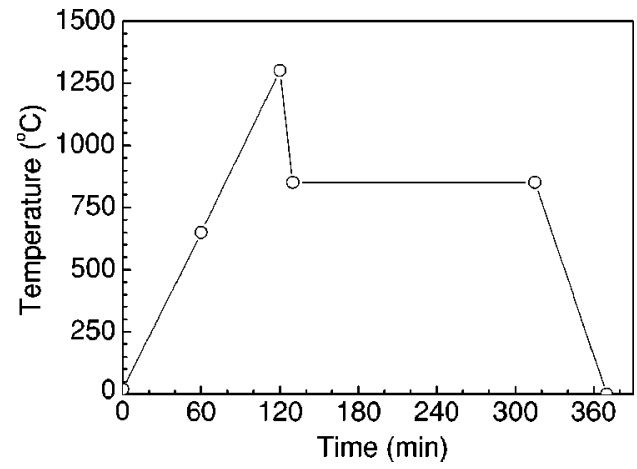

FIG. 1. The temperature regime for the RF melting of the NiMnSb powder and ingot.

obtain spectra where $Z=0$. The fact that in some cases such spectra are obatined suggests that this is not always a significant effect. As Zutic and Valls ${ }^{22}$ point out, $Z$ cannot simply be rescaled due to Fermi velocity mismatch, and hence we do not attempt to do so.

There is also controversy with the PCAR technique due to the quality of fitting of the conductance spectra to the current theoretical model. It has been reported that in certain cases there is no unique solution. ${ }^{24}$ Here we report a study of fit quality as a function of both $P_{t}$ and $Z$, which demonstrates a single minimum in the goodness of fit. This provides a unique solution to the model and an estimation of the error in $P_{t}$. Structural, compositional, magnetic, and electrical characterization of the NiMnSb samples was also carried out in order to better understand the polarization results obtained.

\section{EXPERIMENTAL PROCEDURE}

Two polycrystalline bulk NiMnSb samples were produced using MSR2 RF equipment (Cambridge Ltd., Cambridge, U.K.), from high purity $\mathrm{Ni}(99.8 \%), \mathrm{Mn}(99.9 \%)$, and $\mathrm{Sb}$ $(99.999 \%)$ powders (Alfa Aesar, Johnson \& Matthey, Karlsruhe, Germany) mixed in stoichiometric proportions $1: 1: 1$. The two bulk samples have different preparation procedures prior to $\mathrm{rf}$ melting. One was formed directly from the mixed powder (Prf) and the other was pressed and sintered into an ingot $^{25}$ before melting (Irf). Structural, magnetic, and electrical properties of films made from similar bulk material have been reported elsewhere. ${ }^{26}$ For the melting/ crystallization process, the material was placed into an alumina crucible then developed in argon at atmospheric pressure following the temperature regime in Fig. 1. The stoichiometry of the bulk material was measured using energy dispersive $\mathrm{x}$-ray spectroscopy (EDX) that was performed using an XL $30 \mathrm{ESEM} \mathrm{LaB}_{6}$ (Philips Optique Electronique, Paris, France).

Further treatment of both samples was undertaken to clean the surface and to remove the native oxide layer (temporarily). Wet chemical etching with $10 \%$ hydrofluoric acid (HF) was used for this purpose. Contradictory to previously reported etching of $\mathrm{NiMnSb}$ which shows a constant etch rate, ${ }^{27}$ we find that the etch removes the native oxide and possibly a constituent from the surface of the NiMnSb ma-

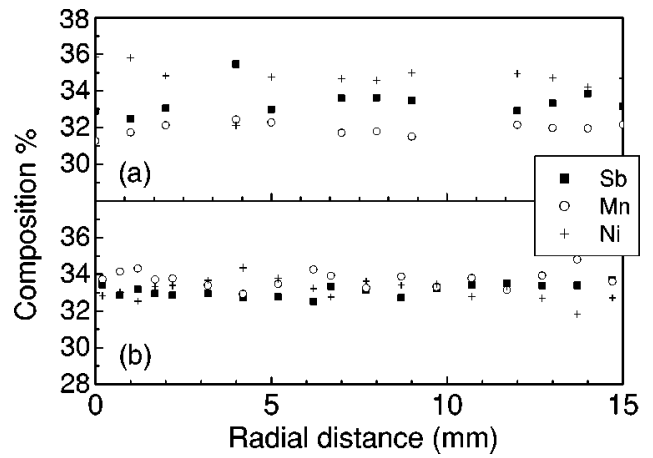

FIG. 2. EDX measurements of the stoichiometric proportions versus radial distance for the (a) PRF and (b) IRF samples.

terial. This leaves a surface that is resistant to further etching, even to $50 \% \mathrm{HF}$ solution. On exposure to air this surface visibly oxidizes within minutes. This fresh oxide layer can then be removed by further etching in HF, which can be used to provide a well controlled etch of NiMnSb. Polarization measurements were taken shortly after etching, but not before surface reoxidization.

Magnetization measurements were performed using an Oxford Instruments vibrating sample magnetometer. Transport spin polarization of the samples was determined using PCAR, ${ }^{14}$ with a superconducting niobium tip which has been formed mechanically. The physical tip size is approximately $5 \times 50 \mu \mathrm{m}$ though from the magnitude of the contact resistance $\left(R_{C}\right)$ the actual contact size $(d)$ is believed to be much smaller. The size of $d$ can be approximated from the general expression $^{28}$

$$
R_{C} \approx \frac{4}{3 \pi} \frac{\rho \lambda}{d^{2}}+\frac{\rho}{2 d},
$$

where $\rho=15 \mu \Omega \mathrm{cm}$ for NiMnSb at $4.2 \mathrm{~K}$ and $\lambda$ is the mean free path. Note that this expression neglects the interface transparency and therefore under estimates $d$. Nevertheless in the present case the data is best fit to the extended BTK model in the ballistic limit meaning that $d \ll \lambda$. For NiMnSb we estimate $\lambda$ to be of the order of $20 \mathrm{~nm}$. Hence although the point contact tip radius is of the order of microns the active geometry must be an assembly of parallel nanocontacts.

All measurements were made with the contact and sample immersed in liquid helium at $4.2 \mathrm{~K}$. The contact resistance (1-200 $\Omega$ ) was measured using a standard four-point configuration. A slowly varying current with a small amplitude ripple was driven through the junction. The differential conductance as a function of the dc bias was obtained by measuring resulting voltage oscillations using a lock-in amplifier.

\section{RESULTS}

EDX measurements of composition shown in Fig. 2 demonstrate that the preparation route has a significant effect on stoichiometry. The pressing and sintering of the $\mathrm{Ni}, \mathrm{Mn}$, and $\mathrm{Sb}$ powder prior to $\mathrm{rf}$ melting forms a bulk material with close to the desired stoichiometric ratio of $1: 1: 1$, Fig. 2(b), 


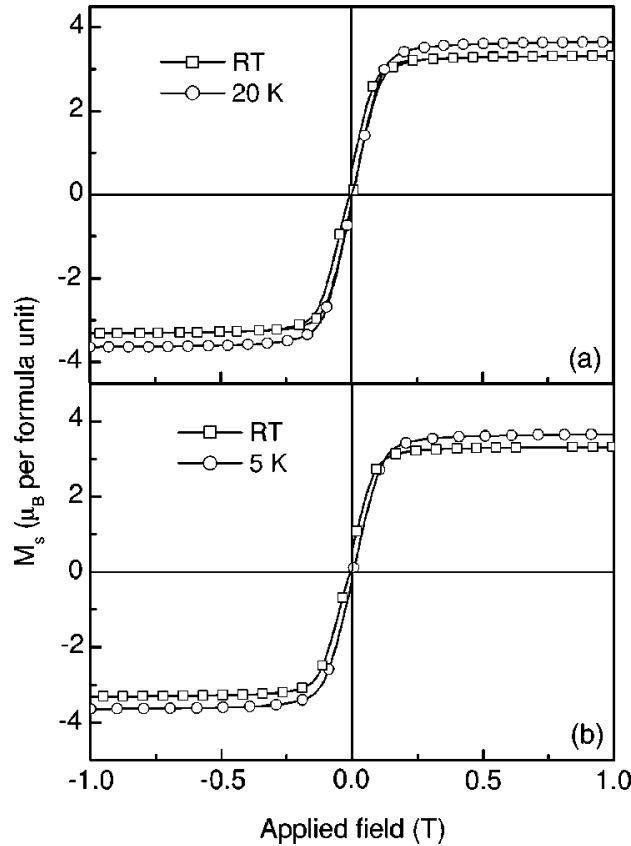

FIG. 3. Magnetic hysteresis loops for the (a) PRF and (b) IRF $\mathrm{NiMnSb}$ samples at low and room temperature.

greatly improved over that of the Prf sample, Fig. 2(a).

Magnetic hysteresis loops for the samples are shown in Fig. 3, at room and low temperature. The low temperature saturation magnetization $\left(M_{s}\right)$ of the samples are almost identical, with an effective moment $\mu_{\text {eff }}=3.60 \pm 0.05 \mu_{B}$ per formula unit. The effective moment falls by $8 \%$ at room temperature to $3.31 \mu_{\mathrm{B}}$. For fully ordered $\mathrm{NiMnSb}$, the predicted $M_{\mathrm{S}}$ should be $4 \mu_{\mathrm{B}}$. X-ray diffraction (XRD) measurements for these samples, which are presented elsewhere, ${ }^{29}$ indicate that about $1 \%$ of second phase NiSb is present in the samples. The reduced saturation magnetization is likely caused by a combination of the presence of the NiSb phase, the off stoichiometry, and the presence of antisite defects. However, it is surprising that the saturation magnetization value is so similar for both samples when there are clear differences in XRD and EDX.

Magnetic force microscopy (MFM) was used to provide information on the domain structure. As shown in Fig. 4, the MFM images of the as-grown Prf and polished Irf samples show striped domain structures with domain periodicity of the order of 1.7 and $4.3 \mu \mathrm{m}$, respectively. We anticipate differences in the interface transparency when the superconducting coherence length is smaller or larger than the domain size and consequently these are also important length scales in the PCAR fitting problem and should always be made explicit. In our case the coherence length for bulk $\mathrm{Nb}$ is of the order of tens of nanometers, and the domain size is of the order of microns.

The differential conductance spectra were normalized to the conductance at high bias $(15 \mathrm{mV})$. These were then modeled using the Mazin extension ${ }^{16}$ to the BTK theory for a S-I-N junction. ${ }^{30}$ There are four fitting parameters; the superconductor energy gap $(\Delta)$, the polarization $\left(P_{t}\right)$, the dimensionless barrier parameter $(Z)$, and a spectral smearing pa-

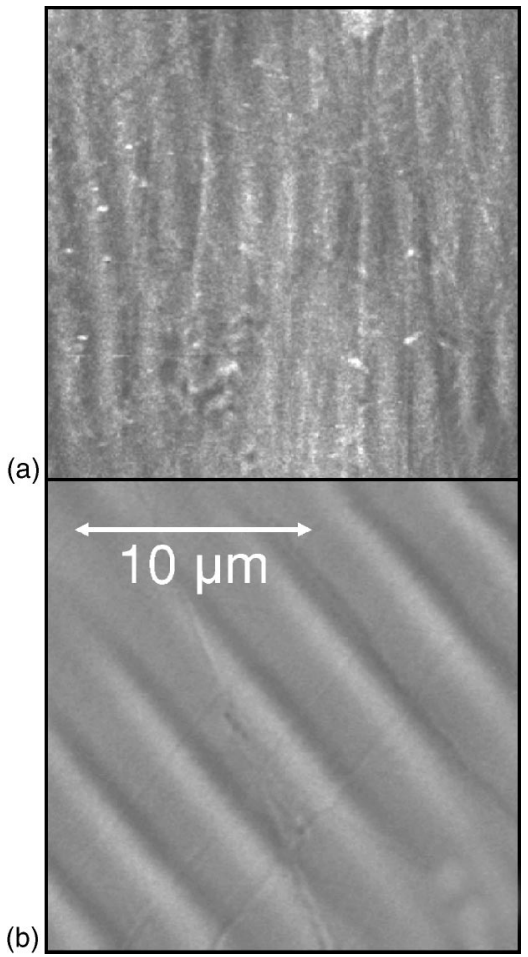

FIG. 4. $20 \mu \mathrm{m} \times 20 \mu \mathrm{m}$ MFM images of the (a) PRF and (b) IRF NiMnSb samples exhibiting striped domain structures with periodicities of 1.7 and $4 \mu \mathrm{m}$, respectively.

rameter $(\Gamma)$. The number of fitting parameters were reduced by discarding data where $\Delta \neq 1.5 \mathrm{meV}$ (the energy gap of $\mathrm{Nb})$.

To illustrate that PCAR can produce unique values of $P$ and $Z$ an iterative quality of fit procedure was carried out for a $\mathrm{Nb}-\mathrm{NiMnSb}$ and a $\mathrm{Nb}$-Co contact which had low and high $Z$ values, respectively. Figure 5 shows the value of $P_{t}$ obtained for the Nb-NiMnSb contact and resulting goodnessof-fit as a function of $Z$. In this way an optimized set of $P_{t}, Z$, and $\Gamma$ parameters were obtained for each conductance spectra. The quality of each fit is shown, with the best fit obtained for $Z=0.05$, with an estimated error in $Z$ of \pm 0.05 . This cor-

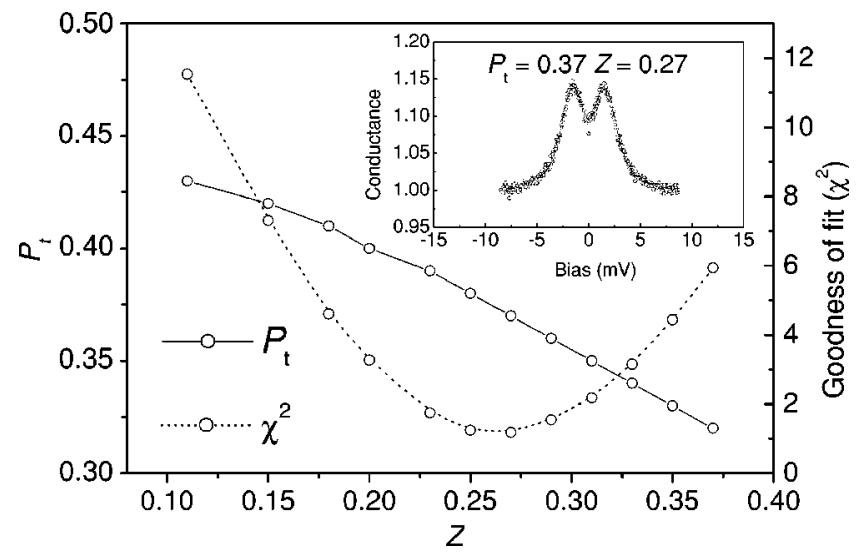

FIG. 5. Fitted polarization $P$ and goodness of fit as a function of $Z$ for a low barrier NiMnSb-Nb contact. Inset: Experimental conductance spectra and best fit when $Z=0.05$ and $P=0.43$. 


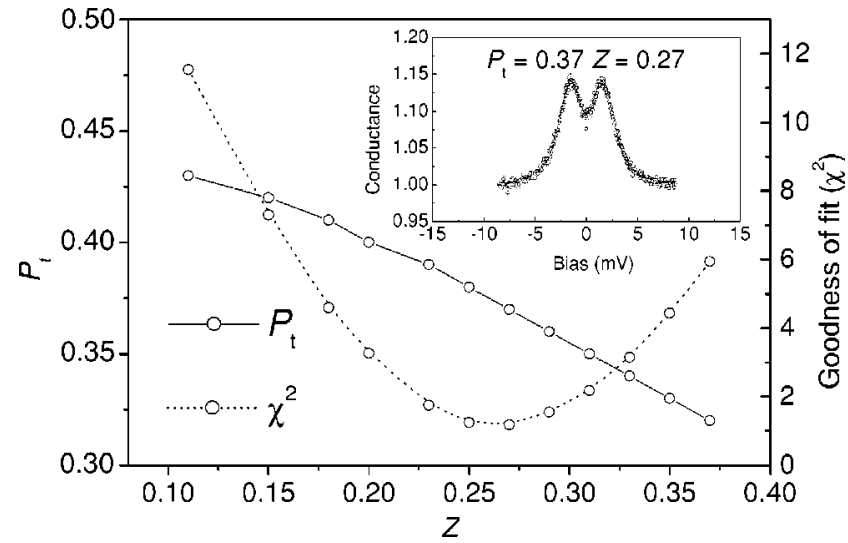

FIG. 6. Fitted polarization $P$ and goodness of fit as a function of $Z$ for a finite barrier $\mathrm{Co}-\mathrm{Nb}$ contact. Inset: Experimental conductance spectra and best fit when $P=0.37$ and $Z=0.27$.

responds to an error in $P_{t}$ of \pm 0.01 . The errors in $P_{t}$ determined from fitting to conductance spectra with high $Z$ values are larger. This is demonstrated by the fitting of a conductance spectrum of a Nb-Co contact with a high Z, Fig. 6. In this example it is clear that the best fit is obtained when $Z$ $=0.27$ with an estimated error in $Z$ of \pm 0.05 . However as there is a greater change in $P_{t}$ with $Z$, the corresponding error in $P_{t}$ is \pm 0.03 . The spectral smearing parameter as obtained from the fitting procedure were consistent between samples and between measurements. Figure 7(b) shows the level of smearing as a funtion of $Z$, and has a typical value of $1 \mathrm{meV}$. This is noticeably larger than the expected value of $0.36 \mathrm{meV}$ for thermal smearing at $4.2 \mathrm{~K}$ [as shown by the dotted line in Fig. 7(b)]. This fact suggests that strong nonthermal broadening mechanisms are at play at the junction interface, presumably associated with interface scattering.

Figure 7(a) shows $P_{t}$ versus $Z$ for both samples with various surface treatments. It is found that there is no discernable difference in $P_{t}$ for the two samples. Etching of the Prf material with HF did not change the value of $P_{t}$ within the estimated error. The decay of $P_{t}$ with increasing $Z$ is seen in all reported PCAR measurements and is probably due to a surface oxide. For both the Prf and Irf samples the polarization is $0.44 \pm 0.05$ at $Z=0$.

\section{DISCUSSION}

From the magnetization measurements of the bulk material we see that the effective moment is slightly below the value of $4 \mu_{\mathrm{B}}$ predicted for half-metallic NiMnSb. ${ }^{31} \mathrm{~A}$ small percentage of atomic disorder may reduce in the ferromagnetic exchange coupling between the local magnetic moments of the $\mathrm{Mn} d$-band and the conduction electrons ${ }^{11}$ bringing $M_{s}$ down. Furthermore electronic structure calculations of disordered $\mathrm{NiMnSb}^{32}$ have shown that small levels of disorder can reduce the minority-spin band gap to zero. Recent theoretical analysis of surface reconstruction at the

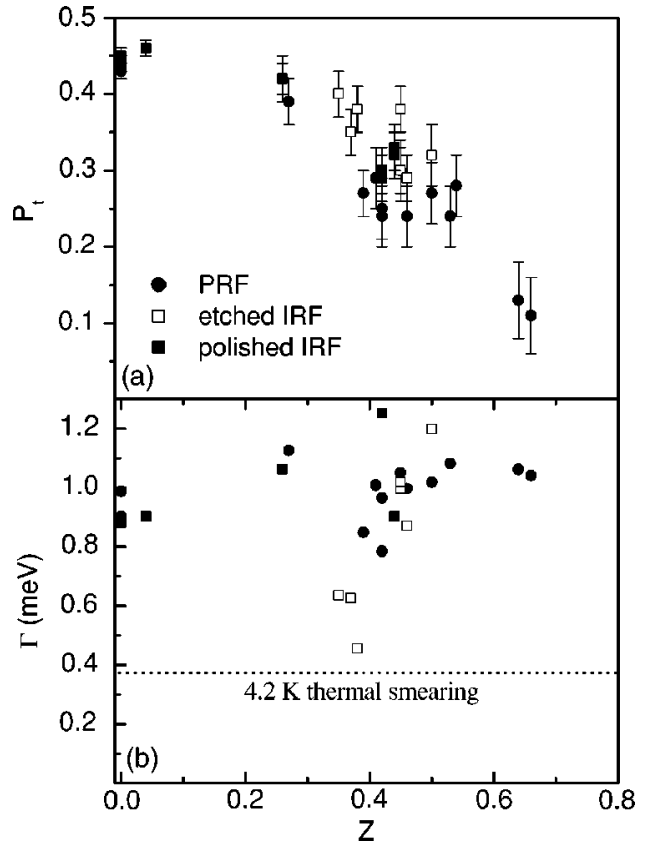

FIG. 7. Measured spin polarization and spectral smearing as a function of $Z$ at $4.2 \mathrm{~K}$ for PRF, polished IRF, and HF etched IRF samples.

free surface of NiMnSb estimates that $P=0 \%$ for Ni terminated surface layers, and $P=30 \%$ in the case of $\mathrm{MnSb}$ termination. ${ }^{33}$ (Angle-resolved photoemission spectroscopy has actually demonstrated the tendency for segregation of $\mathrm{Mn}$ at the free surface of NiMnSb films, ${ }^{34}$ but this result may be specific to films grown on certain substrates.) In the case of the samples studied here, the PCAR measurements were rather insensitive to surface preparation suggesting that it is disorder into the bulk of the material rather than surface reconstruction that is dominating our results. This observation is consistent with the depressed bulk saturation magnetization measurement which indeed ought to correlate with the measured polarization.

\section{CONCLUSION}

From this study it is clear that the two methods of producing bulk NiMnSb described here have little effect on the polarization as measured by PCAR or the global magnetization. It would be desirable to improve the preparation of the material to produce the predicted bulk magnetization of $4 \mu_{\mathrm{B}}$ per formula unit for half-metallic NiMnSb. Matching $\mathrm{NiMnSb}$ films to the tunnel barrier interface while preserving the high spin polarizing properties of the charge carrier is the next significant materials challenge.

\section{ACKNOWLEDGMENT}

This work was supported by the EU Contract FENIKS: G5RD 200100535. 
${ }^{1}$ S. A. Wolf, D. D. Awschalom, R. A. Buhrman, J. M. Daughton, S. von Molnar, M. L. Roukes, A. Y. Chtchelkanova, and D. M. Treger, Science 294, 1488 (2001).

${ }^{2}$ D. D. Awschalom, M. E. Flatte, and N. Samarth, Sci. Am. 286, 66 (2002).

${ }^{3}$ S. Datta and B. Das, Appl. Phys. Lett. 56, 665 (1990).

${ }^{4}$ R. Fiederling, M. Keim, G. Reuscher, W. Ossau, G. Schmidt, A. Waag, and L. W. Molenkamp, Nature (London) 402, 787 (1999).

${ }^{5}$ G. Schmidt and L. W. Molenkamp, Semicond. Sci. Technol. 17, 310 (2002)

${ }^{6}$ E. I. Rashba, Phys. Rev. B 62, R16 267 (2000).

${ }^{7}$ A. Fert and H. Jaffres, Phys. Rev. B 64, 184420 (2001).

${ }^{8}$ V. F. Motsnyi, J. De Boeck, J. Das, W. Van Roy, G. Borghs, E. Goovaerts, and V. I. Safarov, Appl. Phys. Lett. 81, 265 (2002).

${ }^{9}$ A. Hirohata, Y. B. Xu, C. M. Guertler, J. A. C. Bland, and S. N. Holmes, Phys. Rev. B 63, 104425 (2001).

${ }^{10}$ R. A. de Groot, F. M. Mueller, P. G. van Engen, and K. H. J. Buschow, Phys. Rev. Lett. 50, 2024 (1983).

${ }^{11}$ M. J. Otto, H. Feil, R. A. M. Vanwoerden, J. Wijngaard, P. J. Vandervalk, C. F. Vanbruggen, and C. Haas, J. Magn. Magn. Mater. 70, 33 (1987).

${ }^{12}$ G. L. Bona, F. Meier, M. Taborelli, E. Bucher, and P. H. Schmidt, Solid State Commun. 56, 391 (1985).

${ }^{13}$ C. T. Tanaka, J. Nowak, and J. S. Moodera, J. Appl. Phys. 86, 6239 (1999).

${ }^{14}$ R. J. Soulen, J. M. Byers, M. S. Osofsky, B. Nadgorny, T. Ambrose, S. F. Cheng, P. R. Broussard, C. T. Tanaka, J. Nowak, J. S. Moodera, A. Barry, and J. M. D. Coey, Science 282, 85 (1998).

${ }^{15}$ L. Ritchie, G. Xiao, Y. Ji, T. Y. Chen, C. L. Chien, M. Zhang, J. L. Chen, Z. H. Liu, G. H. Wu, and X. X. Zhang, Phys. Rev. B 68, 104430 (2003).

${ }^{16}$ I. I. Mazin, A. A. Golubov, and B. Nadgorny, J. Appl. Phys. 89, 7576 (2001).

${ }^{17}$ Y. Ji, G. J. Strijkers, F. Y. Yang, C. L. Chien, J. M. Byers, A. Anguelouch, G. Xiao, and A. Gupta, Phys. Rev. Lett. 86, 5585 (2001).
${ }^{18}$ B. Nadgorny, M. S. Osofsky, D. J. Singh, G. T. Woods, R. J. Soulen, M. K. Lee, S. D. Bu, and C. B. Eom, Appl. Phys. Lett. 82, 427 (2003).

${ }^{19}$ P. Raychaudhuri, A. P. Mackenzie, J. W. Reiner, and M. R. Beasley, Phys. Rev. B 67, 020411 (2003).

${ }^{20}$ M. S. Osofsky, B. Nadgorny, R. J. Soulen, P. Broussard, M. Rubinstein, J. Byers, G. Laprade, Y. M. Mukovskii, D. Shulyatev, and A. Arsenov, J. Appl. Phys. 85, 5567 (1999).

${ }^{21}$ K. Xia, P. J. Kelly, G. E. W. Bauer, and I. Turek, Phys. Rev. Lett. 89, 166603 (2002).

${ }^{22}$ I. Zutic and O. T. Valls, Phys. Rev. B 61, 1555 (2000).

${ }^{23}$ G. T. Woods, R. J. Soulen, I. I. Mazin, B. Nadgorny, M. Osofsky, J. Sanders, H. Srikanth, W. F. Egelhoff, and R. Datla, cond-mat/ 0307693 (2003).

${ }^{24}$ N. Auth, G. Jakob, T. Block, and C. Felser, Phys. Rev. B 68, 024403 (2003).

${ }^{25}$ A. C. Rowe, Ph.D. thesis, Imperial College, London, 2000.

${ }^{26}$ J. Giapintzakis, C. Grigorescu, A. Klini, A. Manousaki, V. Zorba, J. Androulakis, Z. Viskadourakis, and C. Fotakis, Appl. Phys. Lett. 80, 2716 (2002).

${ }^{27}$ X. A. Cao, J. A. Caballero, K. B. Jung, J. W. Lee, S. Onishi, J. A. Childress, and S. J. Pearton, Solid-State Electron. 42, 1705 (1998).

${ }^{28}$ G. Wexler, Proc. Phys. Soc. London 89, 927 (1966).

${ }^{29}$ C. E. A. Grigorescu, S. A. Manea, M. Mitrea, O. Monnereau, R. Notonier, L. Tortet, R. Keschawarz, J. Giapintzakis, A. Klini, V. Zorba, J. Androulakis, and C. Fotakis, Appl. Surf. Sci. 212, 78 (2003).

${ }^{30}$ G. E. Blonder, M. Tinkham, and T. M. Klapwijk, Phys. Rev. B 25, 4515 (1982).

${ }^{31}$ J. De Boeck, W. Van Roy, J. Das, V. Motsnyi, Z. Liu, L. Lagae, H. Boeve, K. Dessein, and G. Borghs, Semicond. Sci. Technol. 17, 342 (2002).

${ }^{32}$ D. Orgassa, H. Fujiwara, T. C. Schulthess, and W. H. Butler, Phys. Rev. B 60, 13237 (1999).

${ }^{33}$ I. Galanakis, J. Phys.: Condens. Matter 14, 6329 (2002).

${ }^{34}$ D. Ristoiu, J. P. Nozieres, C. N. Borca, B. Borca, and P. A. Dowben, Appl. Phys. Lett. 76, 2349 (2000). 